\title{
Histopathological evaluation of di-ethyl phthalate induced hepatotoxicity on rat
}

\begin{abstract}
Hepatotoxicity in one of the major parameters need to be consider in drug ther-apy, behind the reason that drugs given either in single or in combination for a prolonged period causes liver damage. Most of drug withdraw from the market or pending for approval due to causing hepatotoxicity (Leflunomide, Flutamide, Disulphiram, and Triglitazone) and may be chance to criticize the use based on this toxicity. Hepatoprotective agent opposes this effect while the agent who gen-erates called hepatotoxins. There is no plant in this Universe which is non-me-dicinal and which cannot be made of use for many purposes and by many modes. This definition rightly suggests that in principle all plants have a potential me-dicinal value. Medicinal plants have been considered as important therapeutic aid for alleviating ailment of humankind. The present review is aimed at com-piling data on promising Phytochemical from medicinal plants that have been tested in hepatotoxicity models using modern +scientific system and mechanisms of free radicals toxicity with scavenger which revealed their toxicity belongs to
\end{abstract}

synthetic or herbal product.

Keywords: Liver Diseases; Herbal Treatment; Free Radicals; CYP450

\section{Introduction}

Phthalates are a group of multifunctional chemicals used in plastics, consumer product and medical devices, Despite their wide spread use the toxicity data on these diesters of ophthalmic acid information are limited on pathways of exposure tom phthalates. Diethyl Phthalate was found to be one of the more toxic phthalates. ${ }^{1}$

Diethyl Phthalate is a manufacturing chemical that makes plastic more flexible. It is a phthalic acid ester with chemical formula $\mathrm{C}_{12} \mathrm{H}_{14} \mathrm{O}_{4}$ and commonly identified by CAS registry number -84-66-2 and $\mathrm{LD}_{50}$ value is $8600 \mathrm{mg} / \mathrm{kg}$. It is a colorless liquid with almost no odor. It is everywhere in the environment because of its use in plastic toys, shower curtains adhesives and coatings etc. ${ }^{2}$ A survey of fragrance manufactures conducted in 1995-1996 by the Research Institute for Fragrance Materials reported an annual use of approximately 4000 tones of diethyl phthalate in the preparation of fragrance mixtures. ${ }^{1}$ The route of exposure is through inhalation, dermal, oral and intravenous route. The oral route is the most common way of exposure.

Diethyl Phthalate has been found to injure liver. The liver is one of the common sites of Diethyl Phthalate metabolism and utilization after oral exposure. ${ }^{3}$ Liver is known to be a target site for specific toxins and functions in detoxification and maintaining the metabolic homeostasis of body. The liver is the largest organ in the body and performs an astonishingly large number of tasks that impact all body systems (www.vivo.colostate.edu). It is located in the upper righthand portion of the abdominal cavity, beneath the diaphragm and on top of the stomach, right kidney and intestines. It is coned shaped, dark reddish-brown organ, weighs about 3 pounds (medicalcenter. osu.edu). It consists of hepatocytes and is organized into lobules. It is a metabolic factory, synthesizing and breaking down a verity of substances. When the liver broke down harmful substances its byproducts are excreted into the bile or blood (medicalcenter.osu.edu). Because the body depends on so many liver functions, the liver is also involved in a variety of pathological process.
Volume I Issue 4 - 2015

\author{
Sana Akhtar \\ Toxicologist, Ministry of interior Hospital, Saudi Arabia
}

Correspondence: Sana Akhtar, Toxicologist, Ministry of interior Hospital, Saudi Arabia, Tel 00966537 I00653, Email akhtar_sana@hotmail.com

Received: September 24, 2014 | Published: September 30, 2015
Humans are frequently indirect contact with DEP as chemical is included in numerous cosmetic formulations such as bath products hair sprays, nail polish solvents, shampoos, perfumes after shave lotions and detergents. DEP is known to be released into the environment during its synthesis processes, final utilization of DEP-containing products, or disposal of used products. ${ }^{4}$ The most important release source to the aquatic environment is described to leaching from landfill sites. ${ }^{5}$

Entry of this plasticizer into biological system during transfusions (blood or physiological fluid) or hem dialysis or through food chain and their ubiquitous presence in environment has led to numerous studies on their toxicology. Some phthalates are hepatotoxic, cytotoxic, teratogenic and mutagenic in nature. Certain renal, pulmonary and reproductive dysfunctions have also been reported on exposure to these plasticizers in aquatic invertebrates and mammalian species. ${ }^{6}$

Few investigators have studided the hepatotoxic potential of DEP in a variety of animal species. Acute and chronic administrations of DEP caused variable effects on liver depending upon the species of the animal. ${ }^{7}$ Histopathological examination induced enlarged livers showed fatty vacuolation and congestion followed by a cloudy, swelling excessive fatty regeneration. Dilation of smooth and rough endoplasmic reticulum, mitochondrial swelling and increase in microbes in rat liver have been reported an oral administration for 21days. ${ }^{8}$ Enlargement of cells, dilation of smooth endoplasmic reticulum and changes in hepatic lysosomes of ferrets and swollen mitochondria and increased smooth endoplasmic reticulum in liver of mice. Oral exposure of rats and mice to Diethyl Phthalate result in marked increase in liver mass and increase in relative liver weight. ${ }^{3}$ Hepatic hyperplasia appears to be the initial physiological response to DEHP Morphological changes which were observed in the livers of treated rats included fat deposits in the periportal area. Liver cells became enlarged and lipofuscin deposits accumulated indicating that peroxidation of cellular lipids had occurred. ${ }^{8}$ There was a definite increase in hepatic peroxisomes in the centrilobular and periportal 
areas of the liver and there was often an increase in number of mitochondria. $^{3}$

The present study was done to asses of Diethyl Phthalate, polyvinyl chloride, on rat liver after the 14days sub acute of it. To achieve the aim, we designed our protocol with following objective.

The main objective of the study was to evaluate the Histopathological changes in liver tissue.

\section{Materials and methods}

\section{Experiment protocol}

Ten Healthy male and female Wister Albino rats (body weight 250-300g, age 7-8 weeks) were obtained from the Animal House. Rats were housed in polycarbonate boxes with steel wire tops and rice husk breeding. Rats were given food and water Ad libitum. They were grouped into two $(\mathrm{n}=5)$ :

\section{i. Group I - Control group \\ ii. Group II - Treated group}

The control group animals were exposed to $0.9 \%$ saline by oral gavage. The rats of treated group were orally gavaged with di ethyl phthalate by $840 \mathrm{mg} / \mathrm{kg} /$ day. Animals were exposed for 14 days of duration. Fresh food and water were supplied on daily basis. Individual animals weighted at weekly time interval. During the study period animals were weighted weekly and also observed behavioral and morphological changes. The animals were sacrificed by dose of di ethyl ether on the $15^{\text {th }}$ day of exposure after overnight fasting. The study was approved by the Animal Ethics Committee of department of zoology, according to government norms.

\section{Materials required}

a) Xylene: Absolute alcohol, Different grades of alcohol (70\%, $80 \%, 95 \% \mathrm{v} / \mathrm{v}$ in water), Distilled water, Deionized water.

b) Harris Hematoxylin: Acid alcohol (70\% alcohol, HCL: 100\%)/ Scotts solution.

c) Eosin stain: Blotted paper.

\section{Reagent preparation}

Preparation of Scott's solution: Sodium bicarbonate 2gms, Magnesium sulfate $20 \mathrm{gms}$, Distilled water $1000 \mathrm{ml}$.

Mix well, label, date solution. Recommended for hematoxylin.

Preparation of harris hematoxylin: Haematoxilin crystal $5 \mathrm{gms}$, Alcohol percentage 50ml, Ammo micron or potassium alum $100 \mathrm{gms}$, Distilled water $1000 \mathrm{ml}$, Mercuric oxide red $2.5 \mathrm{gms}$. Hematoxylin is used to differentiate malignant cells from non malignant cells and diagnosis of diseases affecting tissues.

\section{Preparation of liver slide and staining:}

i. Using a smooth edge, glass spreader a maximum of $2 \mathrm{~cm}$ in width make a film of the liver slide $3.5 \mathrm{~cm}$ in length.

ii. Staining and mounting of slides.

iii. Mounting: Section are mounted D.P.X and the cover slip is pressed slightly to drain off excess D.P.X and remove air bubble if any.

iv. Cleaning and labeling stain: Any excess D.P.X is putted the slide after dried the slide one cleaned using absolute alcohol (Table 1).

Table I Reagent time period and action

\begin{tabular}{|c|c|c|c|}
\hline Station & Reagent & Time (mins) & Action of reagent \\
\hline I & Xylene & $2-3$ & To remove the paraffin from tissue \\
\hline 2 & Xylene & $2-3$ & To remove the paraffin from tissue \\
\hline 3 & Ab.Alcohol & 2 & To remove the Xylene \\
\hline 4 & Ab.Alcohol & 2 & To remove the Xylene \\
\hline 5 & $95 \%$ Alcohol & 2 & To remove the Xylene \\
\hline 6 & $70 \%$ Alcohol & 2 & To remove the Xylene \\
\hline 7 & Inhaler & & \\
\hline 8 & Hematoxylin & $10-15$ mins & To remove alcohol stains the nucleus \\
\hline 9 & Tap water & Till excess stain remove & To remove excess stain \\
\hline 10 & Scotts & 2 & Remove excess dyes \\
\hline II & $70 \%$ alcohol & 2 & Remove excess dyes \\
\hline 12 & $95 \%$ Alcohol & 2 & Remove water from the tissue \\
\hline 13 & $95 \%$ Alcohol & 2 & Remove water from the tissue \\
\hline 14 & Eosin & $2-3$ dips & Counter status, stain cytoplasm \\
\hline 15 & $95 \%$ Alcohol & 2 & . \\
\hline 16 & Ab.Alcohol & $2-3$ & $\ldots$ \\
\hline 17 & Xylene & Till liver section cleaned & Clearing agents \\
\hline
\end{tabular}

\section{Results}

After oral exposure of rats with Diethyl Phthalate for 14 days, following results were obtained

\section{Histopathology}

Histopathological evaluation of the terminally sacrificed rats from the treated group did reveal significant microscopic changes in liver. 
Diethyl phthalate exposure at the dose of $840 \mathrm{mg} / \mathrm{kg} / \mathrm{bw} /$ day produced centrilobular necrosis, sinusoid dilation, chronic venous congestions
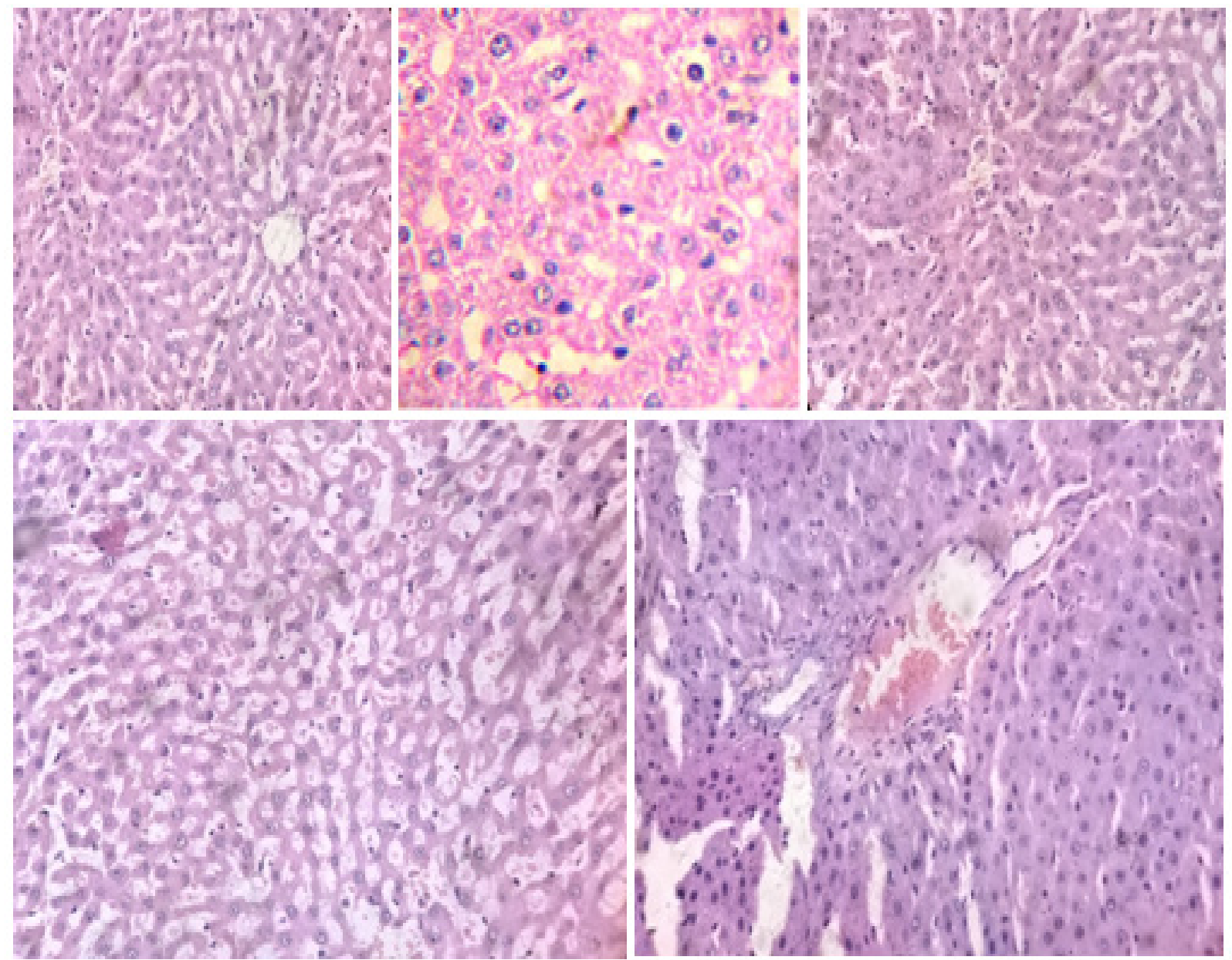

Figure I Normal photomicrograph of liver tissue of control rat showing normal hepatic cells with central vein and sinusoidal dilation ( $\mathrm{H}$ and $\mathrm{E}$ I00X).

\section{Discussion}

There have been a number of studies concerning the potential human health risks from phthalate exposure and diesters of phthalic acid. ${ }^{5}$ Day to day exposure to DEP can result from consuming food stored in plastic packaging which contains DEP, through the usage of a wide array of consumer goods that contain DEP, as a result of medical treatment, i.e., tubing used for dialysis patients and also through occupational exposure (incense stick industry). DEP undergoes degradation in the environment within $72 \mathrm{hrs}$ Lipophilic compounds are highly bioaccumulative in nature as compared to hydrophilic compounds. DEP being lipophilic, major routes of exposure in varying concentrations is through contaminated food or water. ${ }^{9}$

Generally exposure to DEP occurs by daily use of consumer products containing DEP or by occupational exposure at low as well as high concentrations hence usage will be several times a day for a prolonged period.

In this study, we found that sub-chronic exposure to DEP for longer period brings about hepatocellular damage due to oxidative stress (ischemia) leading to increased lysosomal activity, which is evident from a progressive increase in the serum levels of LPO and Catalase and decrease levels of Destructive process in the tissues would lead to leakage of these enzymes into the extra cellular fluids and then into the body fluids which results in the increase in the serum levels of these enzymes. Increased serum levels of these enzymes indicate tissue oxidative stress leading to cell death, which correlates well with the histology.

Hence, DEP given for a prolonged period can lead to hepatocellular damage, which could prove fatal in the future. Significantly increased triglyceride levels in the liver and serum of all the treated groups indicates impairment in the triglyceride metabolism and enhanced triglyceride storage in the liver, which correlates well with the histology and electron micrographs indicating Dose-dependent accumulation of triglycerides in the form of lipid droplets as well as accumulation in mitochondria as observed in electron micrographs may lead to impaired mitochondrial function.

A recent study of dose-dependent toxicity of DEP carried out on male Wistar rat's showed significantly increased serum and liver triglyceride levels in 10,25 and $50 \mathrm{mg} / \mathrm{kg}$ DEP-treated groups. ${ }^{10}$

Liver is the site for synthesis and storage of glycogen, thus being a key organ involved in this metabolic process, increase in the liver glycogen levels would indicate suppressed Carbohydrate metabolism, 
which is clearly seen in the light microscopy especially of the high treated groups. Thus accumulation of excessive glycogen due to DEP treatment at higher doses could lead to hypertrophy of the hepatocytes, which could lead to complete impairment.

Synthesis and metabolism of cholesterol takes place mainly in the liver following which it is transported to other organs. Accumulation of cholesterol in the liver leads to decreases the serum cholesterol levels due to the impairment of cholesterol transport across the hepatocellular membrane. This could lead to impairment in hormone synthesis due to unavailability of cholesterol which is used as a precursor, thus exposure to DEP at higher doses for a prolonged period could lead to impairment of cholesterol transport leading to severe endocrine effects in the reproductive organs. A significant dose-dependent decrease in cholesterol levels in the serum indicates that DEP interferes with cholesterol transport from liver and probably other organs.

\section{Summary}

Diethyl phthalate is used as a plasticizer for cellulose ester plastic films and sheets (photographic) blister packaging and tape applications. Greatest human exposure comes from the use of DEPcontaining consumer products and the ingestion of contaminated foods (seafood, drinking water, or foods contaminated due to DEP leaching from packaging materials). Exposure may also occur via inhalation of contaminated air and from medical treatment involving use of polyvinyl chloride (PVC) tubing. The available studies on the effects of diethyl phthalate are not sufficient.

\section{Acknowledgements}

None.

\section{Conflict of interest}

The author declares no conflict of interest.

\section{References}

1. Hauser R, Calafat AM. Phthalates and Human Health. Occup Environ Med. 2005;62(11):806-818.

2. Bette H. Alert. 2000.

3. David RM. 1999.

4. Giam. Wastes in marine Environments. USA: U.S Government printing office; $1987.130 \mathrm{p}$.

5. Silva MJ, Reidy JA, Herbert AR, et al. Detection of Phthalate Metabolites in Human Amniotic Fluid. Bull Environ Contam Toxicol. 2004;72(6):1226-1231.

6. Thomas JA, Darby TD. A reviews of the biological effects of di-(2ethylhexyl) phthalate. Toxicol Appl Pharmacol. 1978;45(1):1-27.

7. Heena Prajapati, Ramtej J Verma. Nigella sativa ameliorates diethyl phthalate induced lipid infiltration in liver of mice. IJPBS. 2013;3(3):443-449.

8. Lake BG. Young scientist award lecture, Investigations in to the Mechanism of coumarins-induced Hepatotoxicity in Rat. Arch Toxicol. 1983;Suppl 7:16-29.

9. WHO. Concise International Chemical Assessment Document 52. Diethyl Phthalate. 2003.

10. Pereira C, Mapuskar K, Rao CV. Chronic toxicity of diethyl phthalate in male Wistar rats-a dose-response study. Regul Toxicol Pharmacol. 2006;45(2):169-177.++ 